\section{Tips on Examining Metal-Coated Glass Beads for Cracks}

John Twilley, Art Conservation Scientist jtwilley@sprynet.com

I once had a problem looking for cracks in a graphite coating on quartz fibers. This problem is a good example of the general case of defining visible light microscopy inspection criteria. Since specimen lighting may influence critically what one can see. The answer to the question depends a bit on the size of the crack and the opacity and reflectivity of the oxide coated surface. First insure that the microscope has a lighting set up that allows one to dependably see the cracks before worrying about a camera. Most of the digital cameras that can be used with a microscope would probably be adequate to recording the crack, once the viewing situation is set up.

Low magnification approaches include the following:

Try the microscope with lighting as follows: If it has a condenser lens below the sample position, experiment with placing a sheet of something opaque with a small hole in it in the light path so that the light is blocked from passing around the sample. This will give a better chance to see any light passing through the glass bead due to a crack in the coating. Turn off the room lights to get the best view.

Alternatively, one could arrange for "dark field" illumination by doing the opposite: cut some disks (or try some coins in different sizes) and hold them with tweezers in the light path beneath the condenser lens so that light cannot pass through the sample bead directly but must bounce off the sides of the sam- ple to be seen. This may be effective if the bare glass in the cracks is more reflective than the coating.

A $1 \mathrm{~mm}$ round object could be observed well under a stereobinocular microscope common as a "dissecting microscope". This has the advantage of an upright image that will make turning the sphere to view it from all sides easier, and it has good depth of field. However, one would need to arrange the lighting to suit the problem, and if the cracks are very narrow, a magnification exceeding the upper limit of such a microscope would be needed. Some microscopes of this kind are equipped with a ring light, a small fluorescent lamp or a fiber optic fixture around the lens that gives a very diffuse light. This might be helpful if both the bead and the coating are shiny and tend to reflect strongly.

Another low magnification (or no magnificationl) approach might be possible with a fiber optic lamp if one is available and the bead's coating is opaque. Make an opaque cover to fit over the end of the fiber optic with a hole in it that is smaller than $1 \mathrm{~mm}$. It might then be possible to see light projected through a crack when the bead is placed over the hole. Again, use a darkened room to see the light projected through the bead. If this works, orient the fiber optic and bead so that they can be observed with the microscope while doing this.

For very narrow cracks, there may be no alternative to using magnification that is too high to allow one to see the entire bead in focus at one time. In that case, there may be no alternative to racking the focus up and down and systematically rotating the bead to all orientations. If a metallurgical microscope can be found, it will have a simple means of switching between bright field and dark field reflected light. Either could be best, depending on the reflectivities involved.
Acquire,

Process,

E-mail,

Print,

Retrieve

at any time

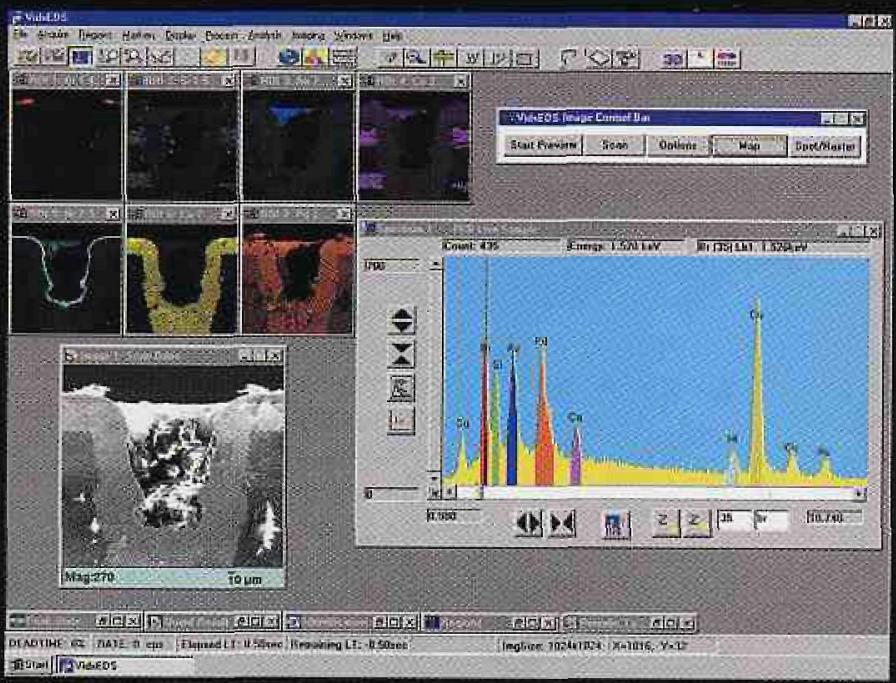

Spectra

Images

Maps

Limescans

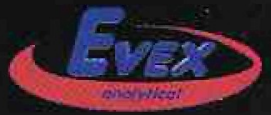

